University of Wollongong

Research Online

Faculty of Engineering and Information

Faculty of Engineering and Information

Sciences - Papers: Part A

Sciences

2007

\title{
Orientation effect on mechanical properties of commercially pure titanium at room temperature
}

\author{
M Battaini \\ Monash University \\ E V. Pereloma \\ University of Wollongong, elenap@uow.edu.au \\ Christopher Davies \\ Monash University
}

Follow this and additional works at: https://ro.uow.edu.au/eispapers

Part of the Engineering Commons, and the Science and Technology Studies Commons

Research Online is the open access institutional repository for the University of Wollongong. For further information contact the UOW Library: research-pubs@uow.edu.au 


\title{
Orientation effect on mechanical properties of commercially pure titanium at room temperature
}

\begin{abstract}
The effect of sample orientation on the mechanical properties of commercially pure (CP) titanium plate with a transverse split basal texture was investigated at room temperature (RT) using plane strain compression (PSC). A large variation in flow stress of up to (EBSD) and calculating Schmid factors for all important slip and twinning modes. Importantly, the Schmid factors were calculated for all orientations in Euler space because there are significant variations over all orientations for the PSC stress state, unlike uniaxial compression or tension. The Schmid factor analysis and twin data for the wide variety of orientations tested enabled the conclusion to be drawn reliably that higher flow stresses were primarily due to an unfavourable orientation for prism- slip. A greater proportion of to twinning was also a major factor in the higher flow stresses. Increased strain hardening was observed in the sample orientation that showed a dramatic texture change to a more difficult orientation for further deformation as a result of dominant twinning. This indicated that reorientation hardening was the responsible mechanism.
\end{abstract}

\section{Keywords}

titanium, properties, mechanical, effect, orientation, room, pure, commercially, temperature

Disciplines

Engineering | Science and Technology Studies

\section{Publication Details}

Battaini, M., Pereloma, E. V. \& Davies, C. H. J. 2007, 'Orientation effect on mechanical properties of commercially pure titanium at room temperature', Metallurgical and Materials Transactions A - Physical Metallurgy and Materials Science, vol. 38A, no. 2, pp. 276-285. 


\title{
Orientation Effect on Mechanical Properties of Commercially Pure Titanium at Room Temperature
}

\begin{abstract}
M. BATTAINI, E.V. PERELOMA, and C.H.J. DAVIES
The effect of sample orientation on the mechanical properties of commercially pure (CP) titanium plate with a transverse split basal texture was investigated at room temperature (RT) using plane strain compression (PSC). A large variation in flow stress of up to $\sim 60$ pct was found for samples of different orientations. The major sources of this variation were revealed by measuring area fractions for individual twin modes using electron backscatter diffraction (EBSD) and calculating Schmid factors for all important slip and twinning modes. Importantly, the Schmid factors were calculated for all orientations in Euler space because there are significant variations over all orientations for the PSC stress state, unlike uniaxial compression or tension. The Schmid factor analysis and twin data for the wide variety of orientations tested enabled the conclusion to be drawn reliably that higher flow stresses were primarily due to an unfavourable orientation for prism- $\langle a\rangle$ slip. A greater proportion of $\{11 \overline{2} 2\}$ to $\{10 \overline{1} 2\}$ twinning was also a major factor in the higher flow stresses. Increased strain hardening was observed in the sample orientation that showed a dramatic texture change to a more difficult orientation for further deformation as a result of dominant $\{10 \overline{1} 2\}$ twinning. This indicated that reorientation hardening was the responsible mechanism.
\end{abstract}

DOI: $10.1007 / \mathrm{s} 11661-006-9040-2$

(C) The Minerals, Metals \& Materials Society and ASM International 2007

\section{INTRODUCTION}

IN common with work on other metals, early literature on deformation mechanisms in titanium looked at the activation of particular slip and twinning modes in suitably oriented single crystals. The critical resolved shear stress (CRSS) for prism- $\langle a\rangle$ and basal- $\langle a\rangle$ slip in titanium was established over a wide range of temperatures and compositions (Conrad ${ }^{[1]}$ for a summary). Prism- $\langle a\rangle$ slip was found to be by far the easiest slip mode up to very high temperatures. However, these deformation modes cannot accommodate a strain component along the $c$-axis. Therefore, pyramidal- $\langle c+a\rangle$ slip and twinning, which are able to accommodate strain along the $c$-axis, are very important.

Similarly to slip, the possible twin modes observed in titanium were determined very early on (the most common modes are shown in Table I), but there is still very little quantitative data on their activity. There have only been a couple of notable studies of twinning in $\alpha$-titanium single crystals. One study ${ }^{[2]}$ found that $\{11 \overline{2} 2\}$ twinning could accommodate almost all the applied strain at room temperature (RT) under favorable loading conditions, indicating its potential as a major deformation mode in polycrystals. The other study $^{[3]}$ determined the resolved shear stress for $\{10 \overline{1} 2\}$ and $\{11 \overline{2} 1\}$ twinning in crystals of three distinct orientations that yielded by either one of these twin

M. BATTAINI, Postgraduate Student, and E.V. PERELOMA and C.H.J. DAVIES, Associate Professors, are with the Victorian Centre for Advanced Materials Manufacturing and the Department of Materials Engineering, Monash University, Victoria 3800 Melbourne, Australia. Contact e-mail: michael.battaini@eng.monash.edu.au

Manuscript submitted March 24, 2006. modes. Comparison of the data showed that a CRSS did not apply for either twin mode, which is in contrast to its use in crystal plasticity modeling as an approximation of an activation criterion. Due to experimental difficulties in activating pyramidal- $\langle\mathrm{c}+\mathrm{a}\rangle$ slip independently, there are very little data on this mode. Unfortunately, this single crystal data may vary enough from polycrystalline data to limit its use, because grain boundaries are obstacles to dislocation motion and may also act as dislocation sources.

A couple of recent studies in polycrystalline titanium have shown that pyramidal- $\langle c+a\rangle$ slip has considerable activity. ${ }^{[4,5]}$ In polycrystals, the twin modes most commonly reported at RT are $\{10 \overline{1} 2\}$ and $\{11 \overline{2} 2\}$ respectively, for deformation perpendicular and parallel to the dominant $c$-axis orientation. ${ }^{[4-10]}$ Other twin modes were found to have high activity under certain conditions. Mullins and Patchett ${ }^{[6]}$ found $\{11 \overline{2} 4\}$ twinning accounted for about one third of all twinning in uniaxial tension along the longitudinal direction of transverse split basal textured sheet. Muruyama ${ }^{[10]}$ also qualitatively observed $\{11 \overline{2} 4\}$ twinning to be a major twin mode in transverse split basal textured sheet. However, this was in plane strain compression (PSC) with compression in the normal direction of the sheet. Glavicic et al. ${ }^{[5]}$ found $\{11 \overline{2} 1\}$ twinning contributed about 30 pet to total twinning in rolling. These data clearly support an orientation dependence of the activated twin mode even if a CRSS does not strictly apply.

Our understanding of the prevalent deformation mechanisms is largely limited by the techniques available for investigation. While several techniques have been used to obtain the required information, each of 
Table I. Twin Mode Parameters for $\alpha$-Ti $(c / a=1.586)$; the Twin Shear was Calculated Using the Formulas in Reference 34

\begin{tabular}{lclll}
\hline $\mathrm{K}_{1}$ & $\eta_{1}$ & Axis & Angle & Shear \\
\hline$\{10 \overline{1} 2\}$ & $\langle 10 \overline{1} \overline{1} \overline{1}\rangle$ & $\langle\overline{1} 2 \overline{1} 0\rangle$ & 84.98 & 0.176 \\
$11 \overline{2} 1\}$ & $\langle 11 \overline{2} \overline{6}\rangle$ & $\langle 1 \overline{1} 00\rangle$ & 34.99 & 0.630 \\
$11 \overline{2} 2\}$ & $\langle 1 \overline{1} \overline{2} \overline{3}\rangle$ & $\langle 1 \overline{1} 00\rangle$ & 64.45 & 0.217 \\
$10 \overline{1} 1\}$ & $\langle 10 \overline{1} \overline{2}\rangle$ & $\langle 1 \overline{2} 10\rangle$ & 57.26 & 0.097 \\
\hline
\end{tabular}

these has its drawbacks. Transmission electron microscopy provides accurate identification but cannot provide statistical information in a realistic time frame. Twin modes may be identified optically by measuring the basal plane trace under polarized light ${ }^{[1,12]}$ or by using an etch pit technique. ${ }^{[13]}$ These two methods are difficult, very time consuming, and can obtain only limited orientation information. Neutron diffraction gives the best statistical representation because it can interrogate a large three-dimensional volume, twin identification can be accurate, and measurements can be made in situ allowing evolution with strain to be tracked. ${ }^{[14,15]}$ The major obstacle with this method is the limited availability of such facilities.

Advances in electron backscatter diffraction (EBSD) and computers have made it possible to achieve accurate twin identification over a large sample area, ${ }^{[16]}$ on equipment that is reasonably accessible. The EBSD provides a large amount of information for quantifying twins, because it contains both a full description of orientation and detailed spatial information over a reasonably large area. ${ }^{[17]}$ This allows orientation relationships between twins and parent grains to be analyzed as well as morphological information. A method for obtaining area fractions of different twins has been described elsewhere in detail ${ }^{[18,19]}$ and will only be presented briefly in Section II of this article.

This article shows how the quantitative twin information obtained from EBSD can be used to relate mechanical properties to texture. The predictions are aided by the calculation of Schmid factors for all orientations, for deformation in PSC, and for all important slip and twinning modes. Together these two pieces of information give a qualitative explanation for the anisotropic flow stresses and strain hardening observed for samples of different orientations. The analysis illustrates the importance of using EBSD as the method for quantifying twinning.

\section{EXPERIMENTAL}

\section{A. Material}

The material used in this study was $10-\mathrm{mm}$ hot-rolled plate of commercially pure $(\mathrm{CP})$, grade 1 , polycrystalline titanium, with impurity limits 0.03 wt pet $\mathrm{N}, 0.08 \mathrm{wt}$ pet $\mathrm{C}, 0.015$ wt pet $\mathrm{H}, 0.20$ wt pet $\mathrm{Fe}$, and 0.18 wt pct $\mathrm{O}$ (ASTM grade 1). The grains in the plate were equiaxed with a mean grain size of approximately $22 \mu \mathrm{m}$. The plate had a typical rolled, transverse-type texture
(Figure 1). The peak intensity of the $c$-axes was tilted away from the normal direction, toward the transverse direction by 30 to $35 \mathrm{deg}$. There was some spread of $c$ axes almost all the way to an alignment parallel to the transverse direction. This texture enabled the orientation effect relative to the important $c$-axis orientation to be determined.

\section{B. Test Method}

Plane strain compression was chosen as the deformation test method because it allows the researcher to isolate the effect on mechanical properties of the orientation of the main texture components. This reduces the deformation to two in-plane directions, which are usually chosen to be aligned along the principal directions of the workpiece from which they are cut: the rolling direction $(R)$, transverse direction $(T)$, and normal direction $(N)$ in the case of the plate in this study. The PSC sample names are then defined relative to this reference frame with the first letter indicating the compression direction and the second letter indicating the extension direction. All of the orientations tested in this study are shown in Figure 2. Their starting textures have been rotated from the plate reference frame into the sample reference for ease of comparison (Figure 4). Sample dimensions were 12$\mathrm{mm}$ length $\times 8$ - $\mathrm{mm}$ height $\times 5.95-\mathrm{mm}$ width, except for the TN sample, which had a length of $10 \mathrm{~mm}$. The PSC tests were performed on a hydraulic Instron (Norwood, MA, USA) at a strain rate of $0.1 \mathrm{~s}^{-1}$ to an equivalent true strain of approximately 0.15 . Displacement was measured by an extensometer clipped onto attachment points on the block and the die of the PSC fittings. A modulus correction was still required to account for

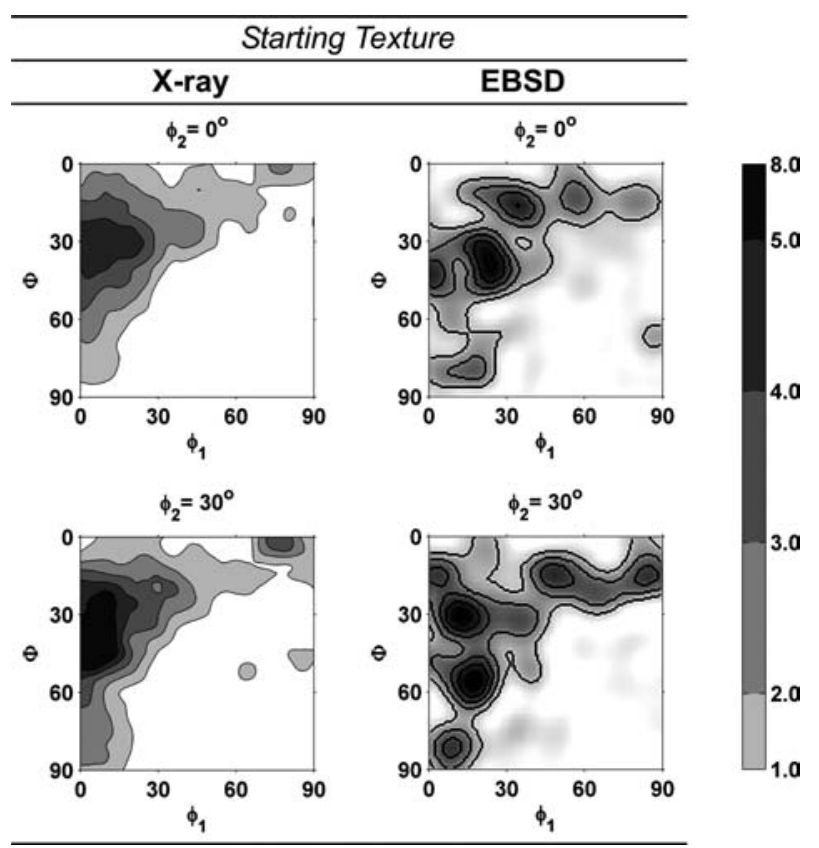

Fig. 1-Comparison of plate starting texture measured by X-ray diffraction and by EBSD. 


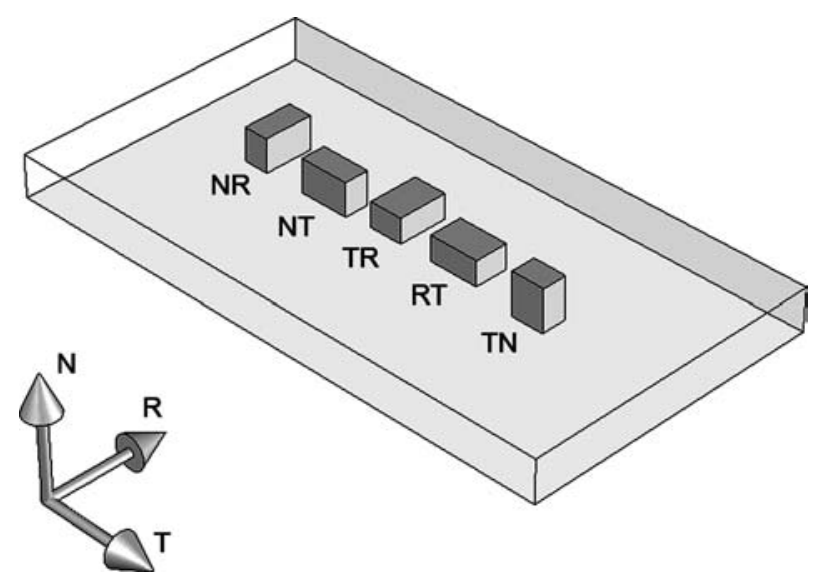

Fig. 2-Orientation and names of plane strain compression samples cut from plate.

the elastic deformation of the PSC fitting tool steel in between the attachment points.

\section{EBSD and X-Ray Texture}

Samples for EBSD and X-ray texture were prepared by mechanically grinding; electropolishing in a solution of $590 \mathrm{~mL}$ methanol, $350 \mathrm{~mL}$ 2-butoxyethanol, and $60 \mathrm{~mL}$ perchloric acid at $\sim 10{ }^{\circ} \mathrm{C}$ for $\sim 10$ seconds at $38 \mathrm{~V}$, and then etching for $\sim 2$ seconds in a solution of $10 \mathrm{~mL} \mathrm{HF}, 30 \mathrm{~mL} \mathrm{HNO}_{3}$, and $50 \mathrm{~mL} \mathrm{H}_{2} \mathrm{O}$.

The EBSD scans were performed over an area of $350 \times 250 \mu \mathrm{m}^{2}$ with a $0.5-\mu \mathrm{m}$ step size on a Leo 1530 Field Emission Gun Scanning Electron Microscope equipped with a Nordlys 2 detector. The scan area gave a good statistical sample of approximately 200 grains. The number of grains analyzed was partially limited by the manual aspect of twin analysis. X-ray texture data were processed using the commercial ResMat software

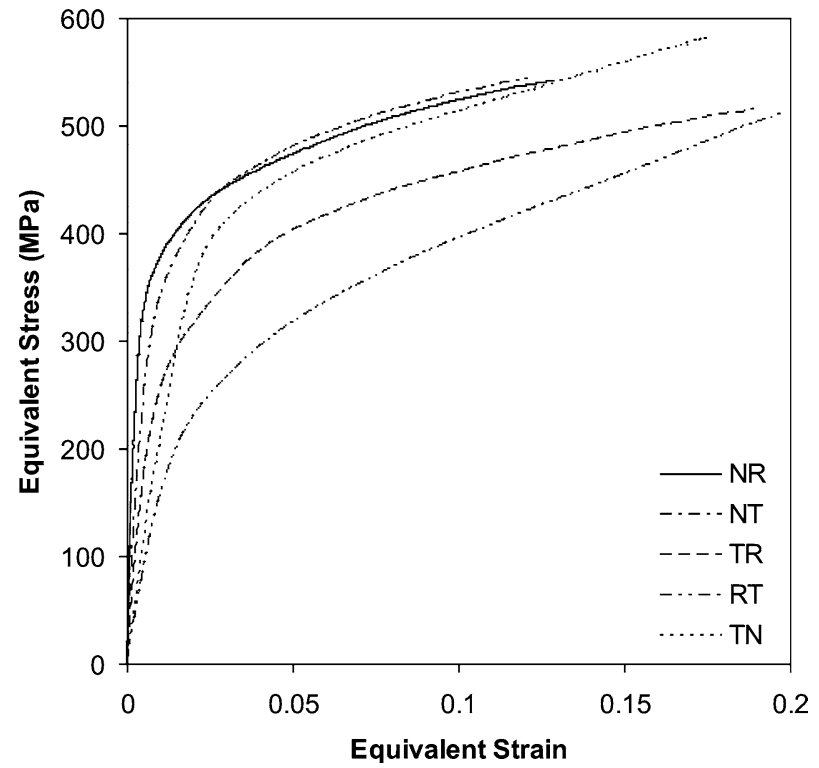

Fig. 3-Modulus-corrected flow curves for plane strain compression of samples of different orientations.
(ResMat Corporation, Montreal, QC, Canada), which uses the spherical harmonics method to calculate the orientation distribution function. Results were plotted using Matlab. HKL Channel 5 software was used for EBSD data collection and analysis. In ResMat, from which the texture data are presented, the crystal reference frame is defined as $\mathrm{X}=[2 \overline{1} \overline{1} 0], \mathrm{Y}=[01 \overline{1} 0]$, and $\mathrm{Z}=[0002]$.

\section{Twin Analysis}

The main information required for determining twin modes from EBSD scans was the misorientation relationship between parent and twin - the axis-angle pair. The axis-angle pairs can be calculated based on the twinning plane $\left(K_{1}\right)$ with a 180 deg rotation and the lattice parameters of the material. Values for the common twin modes in $\alpha$-titanium are shown in Table I. Boundaries following these relationships, to within a 5 deg tolerance, were determined by Channel 5 software. Comparison of the trace of the twin boundary to the trace of the twinning plane in the parent and the twin also provides a check of twin mode. However, this attribute was considered unreliable and was not used in the present analysis, because the exact trace of the boundary plane cannot be determined from a twodimensional EBSD map.

The procedure described is greatly complicated in heavily twinned microstructures because the twins often have a greater area than the parent grain and may even consume the entire grain. This increases the error in parent/twin discrimination. ${ }^{[20]}$ Two other tests were then applied to achieve accurate parent/twin discrimination. First, the orientation of the parent and the twin relative to the applied stress should generally follow a predictable pattern. This can be quantified by the Schmid factor. The potential problem with this approach is that it neglects microscopic deviations from the macroscopic stress. The second test for discrimination of twin regions from parent grains is morphological analysis ${ }^{[19]}$ which is more subjective. In the present study, these last two tests were performed manually. A further consideration was secondary twinning, which was dealt with as follows.

Once a primary twin region forms, it could become favorably oriented for secondary twinning for a different twin mode. This secondary twin could be analyzed in exactly the same way as for the primary twin. In fact, secondary twinning was a minor aid for identifying twinned regions. The secondary twinning always occurred completely within the primary twin. Therefore, the area fraction of primary and secondary twins for each twin mode could be calculated by analyzing EBSD maps with and without the secondary twins filled in. Then, the total area fraction for each twin mode was given as the sum of the primary and secondary twinning for that twin mode.

\section{RESULTS}

The PSC flow curves (Figure 3) showed a very large anisotropy in flow stresses for samples of different 


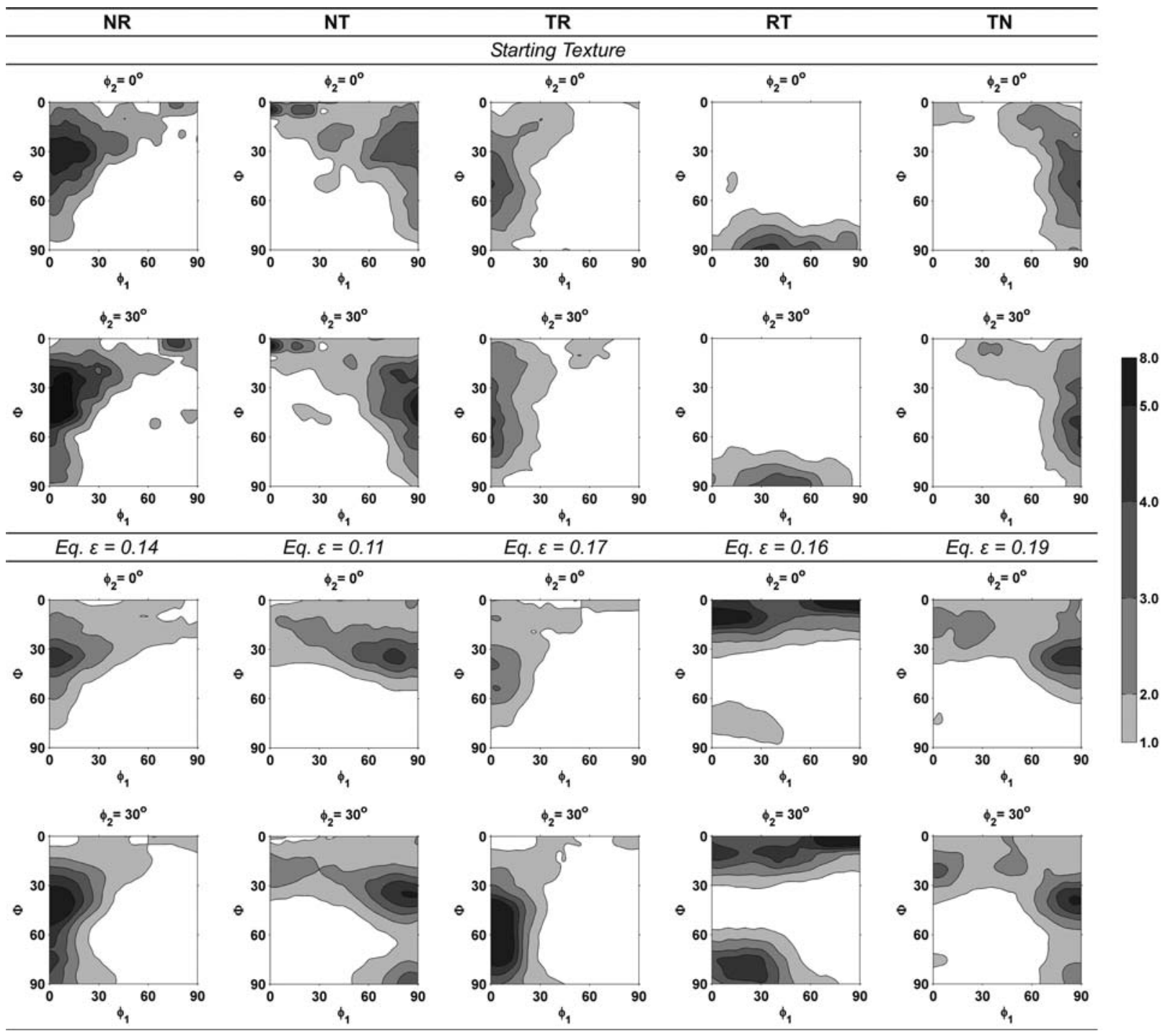

Fig. 4-X-ray textures: the plate starting texture rotated into the sample reference frames is shown in the top row, and the corresponding textures after the given strain are shown in the bottom row. Contour levels are the same for all plots and are shown by the contour bar on the right.

orientations. Samples with the majority of $c$-axes aligned near the compression direction $(\Phi$ values closer to 0 deg), NR and NT (Figure 4), had the highest flow stresses, whereas the sample with the majority of $c$-axes perpendicular to the compression direction $(\Phi$ values closer to $90 \mathrm{deg}$ ), RT, had the lowest flow stresses over the strain range tested, but a noticeably higher hardening rate. The TR sample, with $c$-axis alignment intermediate to these two extremes, had intermediate flow stresses, as might be expected. However, the TN sample also had its main $c$-axis component intermediate to the extremes of texture but had flow stresses comparable to the NR and NT samples. The notable difference between the TN and TR samples is that the $c$-axes are spread toward the constraint direction in the TR sample (along $\varphi_{1}=0 \mathrm{deg}$ ) and toward the extension direction in the TN sample (along $\varphi_{1}=90 \mathrm{deg}$ ). This difference in texture is also observed when the NR and NT samples are compared but without a significant variation in flow stress.

The textures measured after deformation (Figure 4) mostly show very little change, making identification of twinning activity impossible to determine from this information. The RT sample was the only sample that showed a significant change in texture indicative of twinning. The reorientation of the main texture component into alignment with the compression direction (along $\Phi=0 \mathrm{deg}$ ) is distinctive of tensile twinning. Also noticeable but relatively minor in the NT and TN samples was the spread of the main component toward $\varphi_{1}=0 \mathrm{deg}, \Phi=0 \mathrm{deg}$ (more pronounced in the TN sample) and the formation of a component at $\varphi_{1}=90 \mathrm{deg}, \Phi=90 \mathrm{deg}, \varphi_{1}=30 \mathrm{deg}$ (more pronounced in the NT sample). The texture of the NR sample was the least changed by the imposed deformation. 

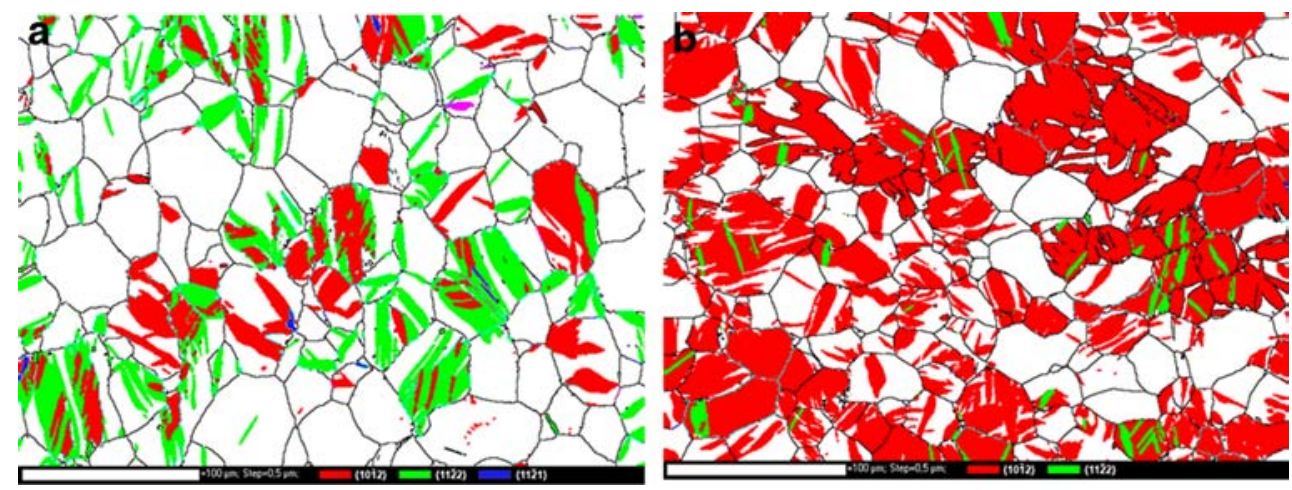

Fig. 5-Example of twin identification from EBSD scans in the (a) NR and (b) RT samples. One small $\{11 \overline{2} 4\}$ twin in the upper-right-hand corner of (a) was omitted from the analysis, because it was the only occurrence and made a negligible contribution to twinning.

Examples of twin identification from EBSD scans are shown in Figure 5. The adequacy of the EBSD scan area was confirmed by comparison of the microtexture to the $\mathrm{X}$-ray texture (Figure 1); the main components were the same but the X-ray texture was smoother, as expected. The area fraction of twinning for each twin mode in each sample was calculated from the EBSD maps (Table II). Note that no $\{10 \overline{1} 1\}$ twinning was found and the contribution of $\{1121\}$ twinning was very small. The component of these values that is made up by secondary twinning is also shown.

The strain accommodated by twinning was calculated from these area fractions using the formula ${ }^{[21]}$

$$
\varepsilon_{\max }=\sqrt{\frac{1}{2}} s V_{\mathrm{twin}}
$$

in which $s$ is the twin shear (given in Table I) and $V_{\text {twin }}$ is the volume fraction of the corresponding twin mode (assumed to be equal to the area fraction for this study). This formula assumes an average orientation of grains in the polycrystal. Note that $\{11 \overline{2} 2\}$ twinning results in a higher strain per unit volume than $\{10 \overline{1} 2\}$, because it has a higher twinning shear. The strains calculated are presented here (Table II) as a percentage of the total strain in the sample as a means of normalization. Values from these calculations show a general trend for a greater contribution to the total strain from $\{11 \overline{2} 2\}$ twinning as the angle of the peak concentration of $c$-axes from the compression direction is decreased. The contribution to the total strain from $\{10 \overline{1} 2\}$ twinning was very large in the RT sample and much lower but still significant in all other samples.

\section{DISCUSSION}

Analysis of twinning by EBSD showed that it contributed a significant percentage of the total strain in all sample orientations. In all but the RT sample, both $\{10 \overline{1} 2\}$ tensile twinning and $\{11 \overline{2} 2\}$ compressive twinning made major contributions. The quantitative results make it clear that the balance between these two twinning modes resulted in an apparently stable deformation texture (Figure. 4). Compression twinning causes a reorientation of grains with their $c$-axis oriented near to the compression direction toward the perpendicular to the compression direction, whereas the opposite is true for tensile twinning, although the rotation angles vary by about $20 \mathrm{deg}$ (Table I). On the other hand, when one twinning mode is dominant, as in the case of the RT sample, a dramatic texture change is observed. These results show the importance of quantifying the incidence and extent of twinning in order to predict the mechanical properties of $\mathrm{CP}$ titanium.

\section{A. Schmid Factor Analysis}

A better understanding of the operation of the different twinning modes and to a certain extent slip modes can be gained by using the Schmid factor to quantify the effect of orientation. This approach was favoured over Taylor factor analysis, because the later introduces assumptions about the CRSS for the different deformation modes and the operative deformation modes. Considering the wide range of CRSS values for hcp deformation modes in the literature, ${ }^{[4,5,22-24]}$ this would unnecessarily complicate the objective of the present analysis. This simple line of analysis has been used to successfully identify twin variants from texture data $^{[25]}$ and to explain the presence of directly observed twin modes. ${ }^{[6,26]}$ Schmid factor diagrams have been produced before for hexagonal metals for the simpler cases of tension and compression. ${ }^{[16,27]}$ However, for these stress states, values do not vary with $\varphi_{1}$. They have also been produced for PSC in diagrams combining all deformation modes for assumed sets of CRSS. $^{[28]}$

In PSC, the Schmid factor varies over all orientations of reduced Euler space. Therefore, the generalized Schmid factor was used:

$$
\begin{gathered}
\mathbf{m}=\operatorname{bg} \boldsymbol{\sigma} \mathbf{g}^{\mathrm{T}} \mathbf{n}^{\mathrm{T}} \\
m=\sum \mathbf{m}
\end{gathered}
$$

in which $\mathbf{b}$ is the slip direction, $\mathbf{g}$ is the orientation matrix, $\sigma$ is the stress tensor, and $n$ is the slip plane. The 
Table II. Area Fraction (AF) in Percent, and Strain Accommodated as a Percentage of Total Strain for Twinning for all Sample Orientations and for Each Twin Mode; the Component of Secondary Twinning out of the Total Values for Each Twin Mode is Also Shown

\begin{tabular}{|c|c|c|c|c|c|c|c|c|c|c|}
\hline \multirow[b]{2}{*}{ Twin } & \multicolumn{2}{|c|}{ NR } & \multicolumn{2}{|c|}{ NT } & \multicolumn{2}{|c|}{ TR } & \multicolumn{2}{|c|}{ RT } & \multicolumn{2}{|c|}{$\mathrm{TN}$} \\
\hline & $\mathrm{AF}$ & Pct $\varepsilon$ & $\mathrm{AF}$ & Pct $\varepsilon$ & $\mathrm{AF}$ & Pct $\varepsilon$ & $\mathrm{AF}$ & Pct $\varepsilon$ & $\mathrm{AF}$ & Pct $\varepsilon$ \\
\hline$\{10 \overline{1} 2\}$ & 11.3 & 10.0 & 14.0 & 15.8 & 14.8 & 10.8 & 48.5 & 36.9 & 24.7 & 16.3 \\
\hline$\{11 \overline{2} 1\}$ & 0.1 & 0.4 & 0.5 & 2.1 & 0.5 & 1.3 & 0 & 0 & 1.4 & 3.2 \\
\hline$\{11 \overline{2} 2\}$ & 18.5 & 20.2 & 25.4 & 35.5 & 16.9 & 15.2 & 2.0 & 1.9 & 15.9 & 13.0 \\
\hline Total & 30.1 & 30.6 & 40.0 & 53.5 & 32.2 & 27.3 & 50.6 & 38.8 & 42.0 & 32.5 \\
\hline \multicolumn{11}{|c|}{ Secondary twinning } \\
\hline$\{1012\}$ & 4.1 & 3.6 & 4.9 & 5.6 & 4.1 & 3.0 & 0 & 0 & 3.6 & 2.4 \\
\hline$\{11 \overline{2} 2\}$ & 4.2 & 4.6 & 5.4 & 7.6 & 4.5 & 4.0 & 2.0 & 1.9 & 4.2 & 3.4 \\
\hline
\end{tabular}

slip plane and direction were first converted to the orthonormal basis from the usual four-index notation used for hexagonal materials. The orientation matrix was calculated from the Euler angles $\left(\varphi_{1}, \Phi, \varphi_{2}\right)$ using the following equation: ${ }^{[29]}$
The NR and NT samples have the highest flow stresses and the highest twin strain percentage for $\{11 \overline{2} 2\}$ twinning. Twinning on $\{11 \overline{2} 2\}$ is expected given the concentration of $c$-axes close to the compression direction (low $\Phi$ values), where Schmid factors are high

$$
\left\{\mathbf{g}=\left[\begin{array}{ccc}
\cos \varphi_{1} \cos \varphi_{2}-\sin \varphi_{1} \sin \varphi_{2} \cos \Phi & \sin \varphi_{1} \cos \varphi_{2}+\cos \varphi_{1} \sin \varphi_{2} \cos \Phi & \sin \varphi_{2} \sin \Phi \\
-\cos \varphi_{1} \sin \varphi_{2}-\sin \varphi_{1} \cos \varphi_{2} \cos \Phi & -\sin \varphi_{1} \sin \varphi_{2}+\cos \varphi_{1} \cos \varphi_{2} \cos \Phi & \cos \varphi_{2} \sin \Phi \\
\sin \varphi_{1} \sin \Phi & -\cos \varphi_{1} \sin \Phi & \cos \Phi
\end{array}\right]\right.
$$

The stress tensor for PSC with compression in the normal direction and extension in the rolling direction was

$$
\sigma=\left[\begin{array}{ccc}
0 & 0 & 0 \\
0 & -0.5 & 0 \\
0 & 0 & -1
\end{array}\right]
$$

The Schmid factor was calculated for all orientations in the reduced Euler space of hexagonal materials. Sections of $\varphi_{2}$ are presented for the main deformation modes in titanium (Figure. 6). The additional constraint of irreversibility of shear sense was applied for twinning. One point to consider when using this type of analysis is that only the favorability of an orientation for a particular mode of deformation is quantified. The actual operation of the deformation modes also depends on the CRSS. Nevertheless, this analysis serves as a good starting point for qualitative assessment of mechanical properties. It may further be used to obtain a lower bound for the yield strength. ${ }^{[30]}$

\section{B. Effect of Orientation on Mechanical Properties}

Comparisons of Schmid factor distributions for twinning (Figure 6) with the starting textures (Figure 4) give a qualitative explanation of the experimental strain accommodation for twinning (Table II) and a trend in the resulting flow stresses (Figure 3). This implies that the activity of twinning is largely dictated by orientation, as illustrated in the following discussion. for this twin mode. There is a substantial percentage of strain accommodated by $\{10 \overline{1} 2\}$ twinning, but more than a third of this is secondary twinning in the favorably oriented $\{11 \overline{2} 2\}$ twins. The remainder of the $\{10 \overline{1} 2\}$ twinning can be accounted for by the spread of texture in these samples toward perpendicular to the compression direction, where Schmid factors are high for this twin mode. As mentioned previously, there is also a variation in Schmid factors with $\varphi_{1}$, although for twinning, the contrast is nowhere near as great as the variation with $\Phi$. Still, there is a concentration of high Schmid factors for both $\{11 \overline{2} 2\}$ and $\{10 \overline{1} 2\}$ at high $\varphi_{1}$ values. This is reflected in the higher twin strain percentages for the NT sample, which has its main texture component at high $\varphi_{1}$ values. The NR sample, which has its main texture component at low $\varphi_{1}$ values, has lower twin strain percentages. However, this did not result in a significant variation in flow stresses between the samples. The reason for this will be explained later in the discussion when slip is addressed.

In contrast to the NR and NT samples, the RT sample has the lowest flow stresses. All the RT sample's primary twinning is the $\{10 \overline{1} 2\}$ mode with only a small amount of secondary $\{11 \overline{2} 2\}$ twinning (Figure 5 (b)). This is also in accord with the high Schmid factors at the high $\Phi$ values at which the main texture component in the RT sample is located. Notably the twinning in the RT sample still accommodates a strain within the range of that for the NR and NT samples.

The TR and TN samples with intermediate orientations to the RT sample and the NR and NT samples have an intermediate strain accommodation by $\{11 \overline{2} 2\}$ twinning. 


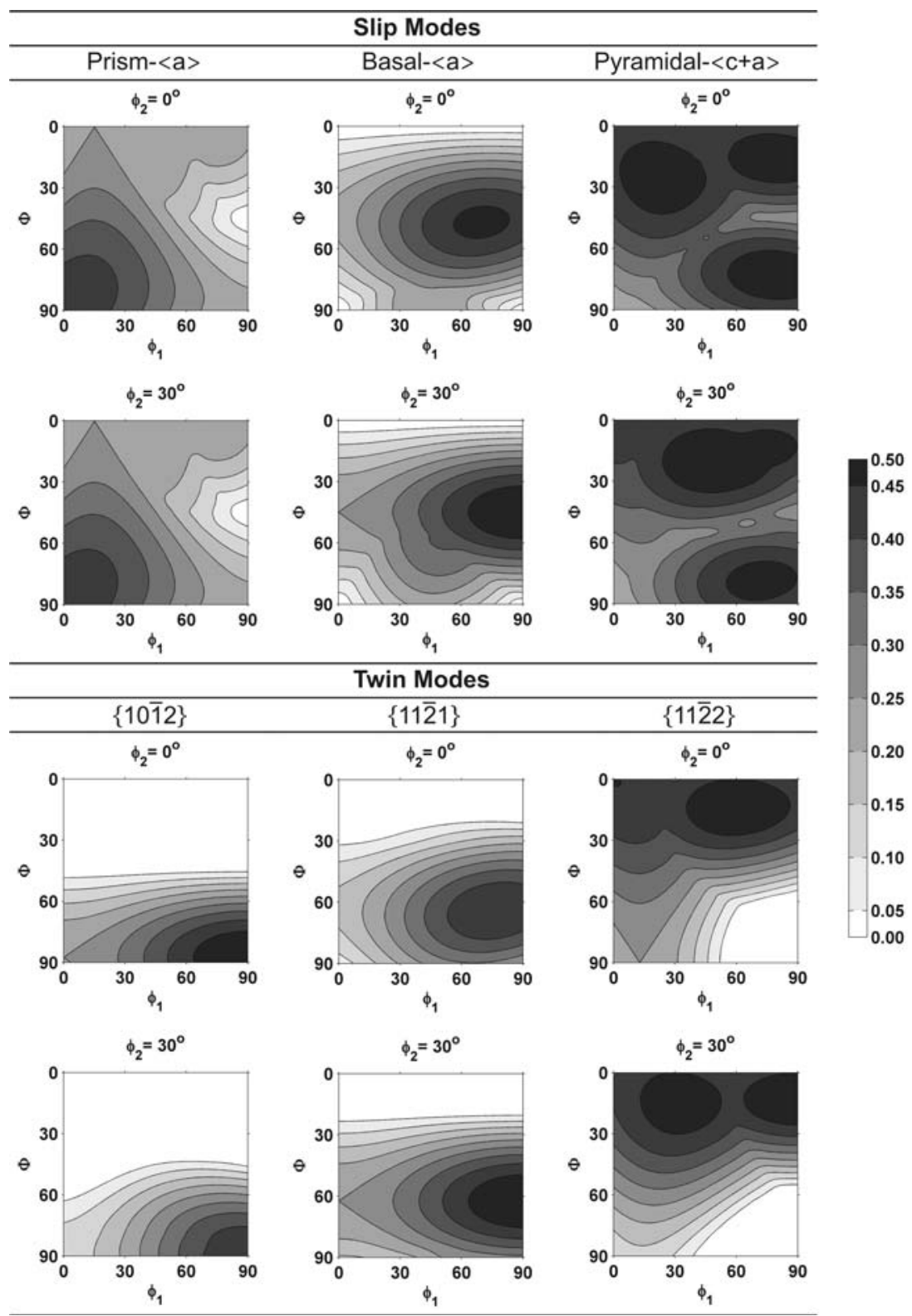

Fig. 6 - Schmid factor Euler space sections for the plane strain compression stress state for the common deformation modes in $\alpha$-Ti.

They also have a large amount of $\{10 \overline{1} 2\}$ twinning but with a smaller contribution from secondary twinning. Continuing the previous trend, the TR sample has intermediate flow stresses. However, the TN sample does not follow this trend-it has much higher flow stresses than the TR sample, comparable to the NR and NT samples. The difference is much greater than would be expected from the increase in Schmid factor for twinning with increase in $\varphi_{1}$ values. This indicates that the Schmid factor for slip is more important than the Schmid factor for twinning, as will be discussed subsequently.

With the quantitative twin data presented, it was straightforward to establish the effect of orientation on the contribution of the different twin modes. Further, these findings roughly correlate to the variation in flow stresses of the different orientation samples - higher flow stresses for samples with more $\{1122\}$ twinning. It appears then that a higher CRSS for $\{11 \overline{2} 2\}$ than $\{10 \overline{1} 2\}$ twinning is a factor in the anisotropic mechanical properties. However, the influence of slip must also be considered. Unfortunately, variation in mechanical properties may similarly be attributed to the variation of slip with orientation as well as twinning. This makes it difficult to determine the effect of slip independently when direct experimental data are not available, Fortunately, the wide variety of orientations tested in this study enable a reasonable, indirect route to be taken.

The key samples that help differentiate the effects of slip from twinning are the TN and TR samples. These 
samples have a very similar strain accommodation from twinning. On close inspection, the ratio of $\{10 \overline{1} 2\}$ to $\{11 \overline{2} 2\}$ twinning is larger in the TN sample. If twinning were the only determinant, this would indicate decreased flow stresses in the TN sample based on the general trend observed for twinning. However, twinning in most of the samples accounts for less than 40 pct of the total strain. Since prism- $\langle a\rangle$ slip is the easiest deformation mode in $\alpha$ $\mathrm{Ti} ;{ }^{[1]}$ it is most likely the other major if not the main contributor to the strain. The TR sample has its main texture component close to the peak in Schmid factors for prism- $\langle a\rangle$ slip (low $\varphi_{1}$ values and high $\Phi$ values). In contrast, the TN sample has its main texture component near the trough in Schmid factors for prism- $\langle a\rangle$ slip (high $\varphi_{1}$ values and intermediate $\Phi$ values). This provides satisfactory evidence that the low probability of prism- $\langle a\rangle$ slip in the TN sample is the main cause of its higher flow stresses compared to the TR sample.

Making this comparison between the RT sample and the NR and NT samples also reveals a stark contrast in terms of propensity for prism- $\langle a\rangle$ slip. The RT sample is the most favorably oriented of any sample for prism- $\langle a\rangle$ slip, whereas prism- $\langle a\rangle$ slip is least likely to occur in the NR and NT samples. It is highly likely, then, that this was a major factor in the anisotropic flow stresses. As discussed previously, the samples are also opposites in terms of twinning: up to 35.5 pct strain accommodation by the more difficult $\{11 \overline{2} 2\}$ twinning mode in the NR and NT compared to only 2 pct in the RT sample (Table II). This suggests that the differences in twinning are also a major factor for the anisotropic flow stresses. However, from the previous observations in the TN and TR samples, it appears that the contrasting propensity for prism- $\langle a\rangle$ slip is the main factor in the anisotropic flow stresses.

The NR and NT samples present a similar contrast to the TN and TR samples in terms of texture about $\varphi_{1}$. When making the same comparison with the prism- $\langle a\rangle$ slip Schmid factor sections, it is surprising then to find that they have similar flow stresses. On this basis, much lower flow stresses would be expected in the NR sample. The important difference between these two pairs of samples, though, is the large discrepancy in the amount of strain accommodated by $\{11 \overline{2} 2\}$ in the NR, NT pair. The contribution is $\sim 76$ pct higher in the NT sample. Because twinning accommodates a strain component along the $c$-axis, prism- $\langle a\rangle$ slip is unlikely to make up a significant amount of this difference. Pyramidal- $\langle c+a\rangle$ slip is the most commonly reported slip mode that can accommodate the $c$-axis strain component. ${ }^{[4,5]}$ Although there is not a large amount of evidence, it seems clear that the CRSS for pyramidal- $\langle\mathrm{c}+\mathrm{a}\rangle$ is relatively high. ${ }^{[4]}$ It then appears that this slip mode makes up the difference in the absence of twinning. This would then explain the comparable flow stresses of the NR and NT samples, the greater ease of prism- $\langle a\rangle$ slip in the NR sample being balanced by the higher activity of pyramidal- $\langle c+a\rangle$ slip.

It is worth mentioning that no $\{11 \overline{2} 1\}$ twinning was observed in the RT sample despite favorable Schmid factors reasonably close to the maximum in this sample. This indicates that this mode is much more difficult to activate than the primary $\{10 \overline{1} 2\}$ tensile twinning mode. The area fractions for $\{11 \overline{2} 1\}$ in the other samples are too small to observe any clear trend, and it may be that the twinning only occurred in highly heterogeneous regions.

The findings on the operation of the different deformation modes generally reflects the flow stresses observed up to the maximum strains measured in this study. All the curves show a strain-hardening rate that rapidly decreases after yielding to a fairly constant rate at a strain of approximately 0.05 (Figure. 3). The one notable difference was a slightly higher strain-hardening rate in the RT sample in the constant strain-hardening region. It suffices to say here, for this small point, that effects from twinning are most likely the source of the difference. $^{[31,32]}$

The mechanisms proposed for strain hardening of $\alpha$ titanium due to twinning are as follows:

(1) a dynamic Hall-Petch effect created by the production of new boundaries from twinning, which decreases the average slip length in the material; ${ }^{[31,32]}$

(2) hardening in the twinned region due to the Basinski mechanism; and ${ }^{[32]}$

(3) reorientation hardening/softening due to the reorientation of twinned regions to a less/more favorable orientation for further deformation. ${ }^{[31,32]}$

Given that the total fraction of twinning is large in all samples, the first two mechanisms should not have a bearing on the differences between samples. The most notable difference in twinning behavior between the RT sample and the other samples is the dominance of one twinning mode- $\{10 \overline{1} 2\}$ twinning (Table II). This resulted in the formation of a strong basal fiber close to the compression direction. As evidenced by the NR and NT samples (Figure 3) and discussed previously in terms of operative deformation modes, this texture requires higher flow stresses for deformation. Therefore, it is likely that the increased hardening in the RT sample is due to reorientation hardening. The low yield stress in this sample makes this more apparent.

\section{Twin Analysis Methods}

The results of the twinning analysis illustrated the importance of using a direct method to determine the activity of twinning. Indirect methods that look simply at the reorientation of texture components would predict little twinning in all but the RT sample. Texture modeling could also be misled into predicting little twinning. Specifically, the small variations in texture could easily be produced by slip rather than by a balance of twinning modes. This point becomes critically important in texture modeling because of the lack of data on the CRSS and hardening parameters for deformation modes in polycrystals. This necessitates an iterative fitting process, whereby parameters are adjusted until the experimental deformation texture and mechanical properties are replicated by the model. If the texture data and mechanical properties from all but the RT sample were used alone for this process, they could be replicated with a set of parameters that greatly favored 
slip over twinning. This last issue highlights two positive aspects of the current data. First, the quantitative twin data reduces the number of variables that have to be fitted by requiring certain twin fractions to be replicated by the model, although it may be better to use cases with a single dominant twin mode such as the RT sample to fit the initial parameters. The second, more practical, benefit is to use the samples with complicated texture evolution as independent tests of a parameter set determined from the simpler cases.

Although laborious, manual twin selection based on twin boundaries satisfying the twin misorientation criteria, orientation, and morphology was straightforward in almost all cases. Parent-twin discrimination was clear from their respective orientations. Grains with their $c$-axes close to parallel (perpendicular) to the compression direction twinned by compressive (tensile) twinning in the majority of cases. Grains with orientations intermediate to these two extremes tended not to twin or, if they did, resulted in twins that consumed only a small fraction of a grain, allowing clear identification based on morphology. Other sources of uncertainty are the twins that could not be identified-which were rare - and poorly defined boundaries, which were minimal with the $0.5 \mu \mathrm{m}$ step size used. It appears that this step size is sufficient for resolving the majority of twin lamellae, at least for $\{10 \overline{1} 2\}$ and $\{11 \overline{2} 2\}$ twinning in titanium. This is supported by easier growth than nucleation of twins ${ }^{[33]}$ together with the relatively large strains used in the present investigation, creating large twin lamellae.

Less obvious and potentially a greater source of uncertainty would be grains fully consumed by twinning. These twins would be unavoidably omitted from the twin fraction resulting in an underestimate of the twinned fraction. However, given the clearly different morphologies of twins and grains, this possibility did not seem to be encountered at the strains used in our experiments. Even the most heavily twinned grains in the RT sample, which appeared close to saturation of twinning, had some segments of twin boundary remaining. Furthermore, the vast majority of grains with the extreme orientations had twins identified within them. This left very few grains that would have been expected to twin but did not or could not be identified as a twin because the grain had been fully consumed by twinning. This may only be a problem at strains well past the saturation of twinning.

Important advantages could be gained from an automated twin analysis procedure by allowing all twin and grain orientations to be interrogated in detail. This may provide data that could not be obtained in a timely manner manually. The present approach favors visual representation for easy manual analysis. Specific orientation information is most relevant in this study to the use of area fraction rather than volume fraction. The area fraction used in the calculation of strain accommodation may be an underestimate of the volume fraction because twins often have a lenticular morphology. Consequently, a twin is unlikely to be sectioned through an axis of symmetry that would allow a direct conversion of area fraction to volume fraction. A more accurate estimate could be made by calculating the true thickness of the twin. This may be done by dividing the apparent area by the sine of the angle between the twin plane and the normal to the section plane and also calculating data for the third dimension, as discussed in Reference 18. This approach may give an improvement but would still not give as precise an estimate as could be obtained from three-dimensional experimental data.

\section{CONCLUSIONS}

EBSD was used to quantify the activity of individual twin modes for meaningful sample areas. The data collected allowed a large number of individual twins to be accurately identified based on crystallographic twin relationships, orientation relative to the applied stress, and morphology. The twin data, together with the Schmid factors calculated - importantly - for all orientations in Euler space for PSC, allowed the following major findings on mechanical properties to be made.

1. Twinning was a major contributor to the total strain in all samples and was almost exclusively the $\{11 \overline{2} 2\}$ and $\{10 \overline{1} 2\}$ modes or the $\{10 \overline{1} 2\}$ mode only.

2. Flow stresses were primarily dictated by the orientation of the main texture component for prism- $\langle a\rangle$ slip-flow stresses increasing as the orientation became less favorable for prism- $\langle a\rangle$ slip.

3. A higher proportion of $\{11 \overline{2} 2\}$ to $\{10 \overline{1} 2\}$ twinning was also a major factor increasing flow stresses.

4. Reorientation hardening became noticeable as a mechanism increasing strain hardening in the sample that had a single dominant twin mode: $\{10 \overline{1} 2\}$ twinning in the RT sample. This resulted in a higher strain-hardening rate than the other samples because the twinned regions had a much less favorable orientation for further deformation. A greater balance between twin modes in the other samples reduced any effect from this strain-hardening mechanism because reorientation hardening was balanced by reorientation softening. Other mechanisms of strain hardening due to twinning were not apparent. This may have been because the total twinning was more constant in all samples, which would reduce the effect from dynamic Hall-Petch hardening and Basinski hardening.

These results quantify the effect of twinning on the behavior of CP titanium in PSC. The twinning data can provide excellent information for validation of modeling results.

\section{ACKNOWLEDGMENTS}

This work was funded by the Victorian Centre for Advanced Materials Manufacturing (VCAMM) and an Australian Postgraduate Award through Monash University. 


\section{REFERENCES}

1. H. Conrad: Progr. Mater. Sci., 1981, vol. 26, pp. 123-493.

2. N.E. Paton and W.A. Backofen: Metall. Trans., 1970, vol. 1, pp. 2839-47.

3. A. Akhtar: Metall. Trans. A, 1975, vol. 6A, pp. 1105-13.

4. S. Zaefferer: Mater. Sci. Eng. A, 2003, vol. 344, pp. 20-30.

5. M.G. Glavicic, A.A. Salem, and S.L. Semiatin: Acta Mater., 2004, vol. 52, pp. 647-55.

6. S. Mullins and B.M. Patchett: Metall. Mater. Trans. A, 1981, vol. 12A, pp. 853-63.

7. Y.B. Chun, S.H. Yu, S.L. Semiatin, and S.K. Hwang: Mater. Sci. Eng. A, 2005, vol. 398, pp. 209-19.

8. A.A. Salem, S.R. Kalidindi, and R.D. Doherty: Acta Mater., 2003, vol. 51, pp. 4225-37.

9. Y. Murayama, K. Obara, and K. Ikeda: Mater. Trans. JIM, 1987, vol. 28, pp. 364-78.

10. Y. Murayama, K. Obara, and K. Ikeda: Mater. Trans. JIM, 1991, vol. 33, pp. 854-61.

11. R.E. Reed-Hill and D.H. Baldwin: Trans. TMS-AIME, 1965 , vol. 233, pp. $842-44$.

12. R.E. Reed-Hill, E.R. Buchanan, and F.W. Caldwell Jr.: Trans. TMS-AIME, 1965, vol. 233, pp. 1716-18.

13. Y. Murayama, K. Obara, and E. Tanaka: Mater. Trans. JIM, 1986, vol. 27, pp. 898-904.

14. P. Rangaswamy, M.A.M. Bourke, D.W. Brown, G.C. Kaschner, R.B. Rogge, M.G. Stout, and C.N. Tomé: Metall. Mater. Trans. A, 2002, vol. 33A, pp. 757-63.

15. D.W. Brown, S.R. Agnew, M.A.M. Bourke, T.M. Holden, S.C. Vogel, and C.N. Tomé: Mater. Sci. Eng. A, 2005, vol. 399, pp. $1-12$.

16. J.F. Bingert, T.A. Mason, G.C. Kaschner, P.J. Maudlin, and G.T. Gray III: Metall. Mater. Trans. A, 2002, vol. 33A, pp. 95563.

17. B.L. Adams, S.I. Wright, and K. Kunze: Metall. Trans. A, 1993, vol. 24A, pp. 819-31
18. T.A. Mason, J.F. Bingert, G.C. Kaschner, S.I. Wright, and R.J. Larsen: Metall. Mater. Trans. A, 2002, vol. 33A, pp. 949-63.

19. B.L. Henrie, T.A. Mason, and J.F. Bingert: ICOTOM-14, Leuven, Belgium, 2005:191-96.

20. B.L. Henrie, T.A. Mason, and B.L. Hansen: Metall. Mater. Trans A, 2004, vol. 35A, pp. 3745-51.

21. S. Mahajan and D.F. Williams: Int. Metall. Rev., 1973, vol. 18, pp. 43-61.

22. S. Myagchilov and P.R. Dawson: Modelling Simul. Mater. Sci. Eng., 1999, vol. 7, pp. 975-1004.

23. J.J. Fundenberger, M.J. Philippe, F. Wagner, and C. Esling: Acta Mater., 1997, vol. 45, pp. 4041-55.

24. N. Cheneau-Spath and J.H. Driver: ICOTOM-10, Clausthal, Germany, 1993, 639-44.

25. S. Godet, L. Jiang, A.A. Luo, and J.J. Jonas: Scripta Mater., 2006, vol. 55 , pp. $1055-58$.

26. M.D. Nave and M.R. Barnett: Scripta Mater., 2004, vol. 51, pp. $881-85$

27. P.G. Partridge: Metall. Rev., 1967, vol. 12, pp. 169-94.

28. F. Xiong and C.H.J. Davies: Magnesium Technol., 2005, 217-22.

29. H.J. Bunge: Texture Analysis in Materials Science: Mathematical Methods, 1st ed., Akademie-Verlag, Berlin, 1982, 21.

30. Y.S. Choi, H.R. Piehler, and A.D. Rollet: Metall. Mater. Trans. A, 2004, vol. 35A, pp. 513-24.

31. A.M. Garde, E. Aigeltinger, and R.E. Reed-Hill: Metall. Trans., 1973, vol. 4, pp. 2461-68.

32. A.A. Salem, S.R. Kalidindi, and S.L. Semiatin: Acta Mater., 2005 , vol. 53, pp. 3495-3502.

33. S.G. Song and G.T. Gray III: Acta Metall. Mater., 1995, vol. 43, pp. 2339-50.

34. J.W. Christian and S. Mahajan: Progr. Mater. Sci., 1995, vol. 39, pp. $1-157$. 